\title{
On Some Three Color Ramsey Numbers for Paths, Cycles, Stripes and Stars
}

\author{
Farideh Khoeini ${ }^{1} \cdot$ Tomasz Dzido $^{2}$
}

Received: 21 July 2017 / Revised: 21 November 2018 / Published online: 4 February 2019

(C) The Author(s) 2019

\begin{abstract}
For given graphs $G_{1}, G_{2}, \ldots, G_{k}, k \geq 2$, the multicolor Ramsey number $R\left(G_{1}, G_{2}, \ldots, G_{k}\right)$ is the smallest integer $n$ such that if we arbitrarily color the edges of the complete graph of order $n$ with $k$ colors, then it contains a monochromatic copy of $G_{i}$ in color $i$, for some $1 \leq i \leq k$. The main result of the paper is a theorem which establishes the connection between the multicolor Ramsey number and the appropriate multicolor bipartite Ramsey number together with the ordinary Ramsey number. The remaining part of the paper consists of a number of corollaries which are derived from the main result and from known results for Ramsey numbers and bipartite Ramsey numbers. We provide some new exact values or generalize known results for multicolor Ramsey numbers of paths, cycles, stripes and stars versus other graphs.
\end{abstract}

Keywords Ramsey number $\cdot$ Bipartite Ramsey number $\cdot$ Cycle $\cdot$ Path $\cdot$ Stripe $\cdot$ Star

Mathematics Subject Classification 05D10 - 05C55

\section{Introduction}

All graphs in this paper are undirected, finite and simple. The union of two graphs $G$ and $H$, denoted by $G \cup H$, is a graph with vertex set $V(G) \cup V(H)$ and edge set $E(G) \cup E(H)$. The join of two graphs $G$ and $H$, denoted by $G+H$, is a graph with vertex set $V(G) \cup V(H)$ and edge set $E(G) \cup E(H) \cup\{u v \mid u \in V(G), v \in V(H)\}$.

Tomasz Dzido

tdz@inf.ug.edu.pl

Farideh Khoeini

f.khoeini@math.iut.ac.ir

1 Department of Mathematical Sciences, Isfahan University of Technology, Isfahan, Iran

2 Institute of Informatics, Faculty of Mathematics, Physics and Informatics, University of Gdańsk, 80-308 Gdańsk, Poland 
The union of $k$ disjoint copies of the same graph $G$ is denoted by $k G$. $\bar{G}$ stands for the complement of the graph $G$. We denote by $G[U]$ the subgraph of $G$ induced by the vertex set $U$. By $P_{n}$ and $C_{n}$ we denote the path and cycle on $n$ vertices, respectively. For a 3-edge coloring (say blue, red and green) of a graph $G$, we denote by $G^{b}\left(G^{r}\right.$ and $G^{g}$ ) the subgraph induced by the edges of color blue (red and green, respectively).

For given graphs $G_{1}, G_{2}, \ldots, G_{k}, k \geq 2$, the multicolor Ramsey number $R\left(G_{1}, G_{2}, \ldots, G_{k}\right)$ is the smallest integer $n$ such that if we arbitrarily color the edges of the complete graph of order $n$ with $k$ colors, then it contains a monochromatic copy of $G_{i}$ in color $i$, for some $1 \leq i \leq k$. The existence of such a positive integer is guaranteed by Ramsey's classical result [17]. Ramsey numbers are still in the main stream of investigations and there are no many results in the field of multicolor and even three-color Ramsey numbers. There are a lot of open cases (see [18]).

The bipartite Ramsey number $b\left(G_{1}, \ldots, G_{k}\right)$ is the smallest positive integer $b$ such that any coloring of the edges of $K_{b, b}$ with $k$ colors contains a monochromatic copy of bipartite $G_{i}$ in the $i$-th color, for $i, 1 \leq i \leq k$.

In 2005, the second author began to determine the exact values for three color Ramsey numbers for two paths and one cycle. He proved that for $n \geq 6, R\left(P_{3}, P_{3}, C_{n}\right)=n$ and $R\left(P_{3}, P_{4}, C_{n}\right)=n+1$ [4]. In 2006 Dzido et al. [6] proved that $R\left(P_{4}, P_{4}, C_{n}\right)=$ $n+2$ and $R\left(P_{3}, P_{5}, C_{n}\right)=n+1$. In 2009 Dzido and Fidytek [5] (and independently Bielak in [2]) obtained the exact value of $R\left(P_{i}, P_{k}, C_{m}\right)$ for several values of $i, k$ and $m$.

Theorem 1 [2,5] Let $i, k, m$ be integers such that $m \geq 3$ is odd, $k \geq m$, and $k>$ $\frac{3 i^{2}-14 i+25}{4}$ when $i$ is odd, and $k>\frac{3 i^{2}-10 i+20}{8}$ when $i$ is even. Then

$$
R\left(P_{i}, P_{k}, C_{m}\right)=2 k+2\left\lfloor\frac{i}{2}\right\rfloor-3 .
$$

In Sect. 3.1 we extend this result to other conditions on the length of paths and a cycle. By a short proof we show that for integers $n_{0}, n_{1}, n_{2}$ such that $n_{0}$ is sufficiently large, if $n_{1}=2 s$ and $n_{2}=2 m$ such that $m-1<2 s$, or $n_{1}=n_{2}=2 s$ or $n_{1}=2 s+1$ and $n_{2}=2 m$ such that $s<m-1<2 s+1$ then

$$
R\left(C_{n_{0}}, P_{n_{1}}, P_{n_{2}}\right)=n_{0}+\left\lfloor\frac{n_{1}}{2}\right\rfloor+\left\lfloor\frac{n_{2}}{2}\right\rfloor-2 .
$$

It should be noted that this result can be also obtained as a consequence of Theorem 2.2 in [14].

For the Ramsey number of paths a well-known theorem of Gerencsér and Gyárfás [10] states that $R\left(P_{n}, P_{m}\right)=m+\lfloor n / 2\rfloor-1$ where $m \geq n \geq 2$. In 1975 [7] Faudree and Schelp determined $R\left(P_{n_{1}}, P_{n_{2}}, P_{n_{3}}\right)$ for the case $n_{1} \geq 6\left(n_{2}+n_{3}\right)^{2}$ and they conjectured that

$$
R\left(P_{n}, P_{n}, P_{n}\right)= \begin{cases}2 n-2 & \text { if } n \text { is even } \\ 2 n-1 & \text { if } n \text { is odd }\end{cases}
$$


In 2007 this conjecture was established by Gyárfás et al. [11] for sufficiently large $n$. We can apply our result for $R\left(C_{n_{0}}, P_{n_{1}}, P_{n_{2}}\right)$ to $P_{n_{0}}$ instead of $C_{n_{0}}$ to obtain the same result as in [7]. The formula is the same but the original result contains the lower bound for $n_{0}$ and no conditions for $n_{1}$ and $n_{2}$ (except $n_{1}, n_{2} \geq 2$ ).

The Ramsey number of a star versus a path was completely determined by Parsons [15]. In Sects. 3.3 and 3.6 we investigate multicolor Ramsey number of a cycle $C_{n}$ or path $P_{n}$ versus stars and stripes for large value of $n$.

In [13] Maherani et al. proved that $R\left(P_{3}, k K_{2}, t K_{2}\right)=2 k+t-1$ for $k \geq t \geq 3$. In this paper we show that $R\left(P_{n}, k K_{2}, t K_{2}\right)=n+k+t-2$ for large $n$. In addition we prove that for even $k, R\left((k-1) K_{2}, P_{k}, P_{k}\right)=3 k-4$. For $s<m-1<2 s+1$ and $t \geq m+s-1$, we obtain that $R\left(t K_{2}, P_{2 s+1}, P_{2 m}\right)=s+m+2 t-2$.

We also provide some new exact values or generalize known results for other multicolor Ramsey numbers of paths, cycles, stripes and stars versus other graphs.

\section{Main Results}

Theorem 2 For every graph $H$ and bipartite graphs $G_{1}, \ldots, G_{k}$, we have

$$
R\left(H, G_{1}, \ldots, G_{k}\right) \leq R\left(H, K_{b, b}\right),
$$

where $b=b\left(G_{1}, \ldots, G_{k}\right)$.

Proof Assume $R\left(H, K_{b, b}\right)=n$, we will show that for any coloring of the edges of the complete graph $K_{n}$ by $k+1$ colors there exists a color $i$ for which the corresponding color class contains $G_{i}$ as a subgraph.

Suppose that $G=K_{n}$ is $(k+1)$-edge colored such that $G$ does not contain $H$ of color 1 . We show that there is a copy of $G_{i}$ of color $i$ in $G$ for some $2 \leq i \leq k+1$. Now we merge $k$ colors classes $2, \ldots, k+1$. Suppose that new class is black. Since $R\left(H, K_{b, b}\right)=n$, we have a black copy of $K_{b, b}$. Using to its original $k$-coloring, we see that there exists a complete bipartite subgraph $L=K_{b, b}$ whose edges are colored with $2, \ldots, k+1$ (observe that there is no edges of color 1 in $L$ ). Now $b=b\left(G_{1}, \ldots, G_{k}\right.$ ) guarantees that $L$ contains a copy of $G_{i}$ of color $i$ for some $2 \leq i \leq k+1$.

Häggkvist [12] obtained the upper bound $R\left(P_{m}, K_{n, k}\right) \leq k+n+m-2$. In addition, Faudree et al. [9] obtained the exact value $R\left(t K_{2}, K_{n, n}\right)=\max \{n+2 t-1,2 n+t-1\}$. Using these results with Theorem 2 we immediately obtain the following.

Corollary 1 For bipartite graphs $G_{1}, \ldots, G_{k}$, we have

1.

$$
R\left(P_{m}, G_{1}, \ldots, G_{k}\right) \leq 2 b+m-2,
$$


2.

$$
R\left(t K_{2}, G_{1}, \ldots, G_{k}\right) \leq \begin{cases}2 b+t-1 & \text { if } t \leq b \\ b+2 t-1 & \text { if } t \geq b\end{cases}
$$

where $b=b\left(G_{1}, \ldots, G_{k}\right)$.

For bipartite graphs $G_{1}, G_{2}, \ldots, G_{k}$ we easily have $R\left(G_{1}, G_{2}, \ldots, G_{k}\right) \leq$ $2 b\left(G_{1}, G_{2}, \ldots, G_{k}\right)$. The answer to the question when the equality holds is an open problem. For example, we know that $b\left(C_{2 m}, C_{4}\right)=m+1$ for $m \geq 4$, while $R\left(C_{2 m}, C_{4}\right)=2 m+1$ for $m \geq 3$ (see [18,19], respectively).

Corollary 2 If $R\left(G_{1}, G_{2}, \ldots, G_{k}\right)=2 b\left(G_{1}, G_{2}, \ldots, G_{k}\right)=2 t$ for bipartite graphs $G_{1}, \ldots, G_{k}$, then

$$
R\left(t K_{2}, G_{1}, \ldots, G_{k}\right)=3 t-1
$$

Proof By Corollary 1, the upper bound is clear. To see the lower bound consider the graph $G=K_{3 t-2}=K_{2 t-1}+K_{t-1}$. Since $R\left(G_{1}, \ldots, G_{k}\right)=2 t$, we take a $k$-coloring of $E\left(K_{2 t-1}\right)$ which does not contain a copy of $G_{i}$ in color $i$ for any $1 \leq i \leq k$. The remaining edges of $G$ we color with color $(k+1)$ and clearly such $G^{k+1}$ contains no copy of $t K_{2}$. This proves the corollary.

\section{More Corollaries}

This section contains a number of corollaries following from the main Theorem 2 and some known results about Ramsey numbers and bipartite Ramsey numbers.

\section{1 $R\left(C_{n_{0}}, P_{n_{1}}, P_{n_{2}}\right)$ for Large $n_{0}$}

In this subsection, we determine the value of $R\left(C_{n_{0}}, P_{n_{1}}, P_{n_{2}}\right)$ for large $n_{0}$ and special cases of $n_{1}, n_{2}$. We first recall a result of Bondy and Erdős from 1973 [1] for $n>n_{1}(r)$ (that is for sufficiently large $n$ ). More precisely, they showed the following.

Theorem 3 [1] For $n>n_{1}(r, t), R\left(C_{n}, K_{r}^{t+1}\right)=t(n-1)+r$ where $K_{r}^{t+1}$ is the complete $(t+1)$-partite graph with parts of size $r$. In particular $R\left(C_{n}, K_{r, r}\right)=n+r-1$.

We can apply this result and Theorem 2 to determine the value which can be also obtained as consequence of Theorem 2.2 in [14].

Theorem 4 For sufficiently large $n_{0}$, if $n_{1}=2 s$ and $n_{2}=2 m$ such that $m-1<2 s$ or if $n_{1}=n_{2}=2 s$ or if $n_{1}=2 s+1$ and $n_{2}=2 m$ such that $s<m-1<2 s+1$, then we have

$$
R\left(C_{n_{0}}, P_{n_{1}}, P_{n_{2}}\right)=n_{0}+\left\lfloor\frac{n_{1}}{2}\right\rfloor+\left\lfloor\frac{n_{2}}{2}\right\rfloor-2 .
$$


Proof For the upper bound, by Theorem 2, $R\left(C_{n_{0}}, P_{n_{1}}, P_{n_{2}}\right) \leq R\left(C_{n_{0}}, K_{b, b}\right)$ where $b=b\left(P_{n_{1}}, P_{n_{2}}\right)$. By Theorem 3, $R\left(C_{n_{0}}, P_{n_{1}}, P_{n_{2}}\right) \leq n_{0}+b-1$. On the other hand, $b=\left\lfloor\frac{n_{1}}{2}\right\rfloor+\left\lfloor\frac{n_{2}}{2}\right\rfloor-1$ (see Theorem 5) and $R\left(C_{n_{0}}, P_{n_{1}}, P_{n_{2}}\right) \leq n_{0}+\left\lfloor\frac{n_{1}}{2}\right\rfloor+\left\lfloor\frac{n_{2}}{2}\right\rfloor-2$.

For the lower bound, consider the graph $G=K_{R-1} \cup K_{\frac{n_{2}}{2}-1}$ where $R=$ $R\left(C_{n_{0}}, P_{n_{1}}\right)$. It is known that $R=n_{0}+\left\lfloor\frac{n_{1}}{2}\right\rfloor-1$ for $n_{0} \geq n_{1} \geq 2$. It is clear that there is a blue/red coloring of $K_{R-1}$ such that $G^{b}$ contains no copy of $C_{n_{0}}$ and $G^{r}$ contains no copy of $P_{n_{1}}$. Color the remaining subgraph $K_{\frac{n_{2}}{2}-1}$ with red. Since $\frac{n_{2}}{2}-1<n_{1}$, there is no a red copy of $P_{n_{1}}$ in $K_{\frac{n_{2}}{2}-1}$. Consider $\stackrel{\bar{G}}{=} \bar{K}_{R-1}+\bar{K}_{\frac{n_{2}}{2}-1}$ and color it with green. Thus $G^{g}$ contains no copy of $P_{n_{2}}$. The equality follows.

In 1975 Faudree and Schelp [7] proved that if $n_{0} \geq 6\left(n_{1}+n_{2}\right)^{2}$, then $R\left(P_{n_{0}}, P_{n_{1}}, P_{n_{2}}\right)=n_{0}+\left\lfloor n_{1} / 2\right\rfloor+\left\lfloor n_{2} / 2\right\rfloor-2$ for $n_{1}, n_{2} \geq 2$. Since $R\left(P_{n_{0}}, P_{n_{1}}, P_{n_{2}}\right) \leq$ $R\left(C_{n_{0}}, P_{n_{1}}, P_{n_{2}}\right)$, we can apply Theorem 4 to $P_{n_{0}}$ instead of $C_{n_{0}}$ to obtain the same results as in [7].

Corollary 3 For sufficiently large $n_{0}$, if $n_{1}=2 s$ and $n_{2}=2 m$ where $m-1<2 s$ or if $n_{1}=n_{2}=2 s$ or if $n_{1}=2 s+1$ and $n_{2}=2 m$ where $s<m-1<2 s+1$, then we have

$$
R\left(P_{n_{0}}, P_{n_{1}}, P_{n_{2}}\right)=n_{0}+\left\lfloor\frac{n_{1}}{2}\right\rfloor+\left\lfloor\frac{n_{2}}{2}\right\rfloor-2 .
$$

Proof Since $R\left(P_{n_{0}}, P_{n_{1}}\right)=n_{0}+\left\lfloor\frac{n_{1}}{2}\right\rfloor-1$ for $n_{0} \geq n_{1} \geq 2$, let us consider the same graph and coloring as in the proof of Theorem 4.

\section{$3.2 R\left(t K_{2}, P_{k}, P_{k^{\prime}}\right)$}

In 1975 Faudree and Schelp [8] determined $b\left(P_{n}, P_{k}\right)$ for all $n$ and $k$. In the following theorem we present only two cases which we will use in the proof of the next theorem.

Theorem 5 [8] For $s, m$ positive integers,

1. $b\left(P_{2 s}, P_{2 m}\right)=s+m-1$,

2. $b\left(P_{2 s+1}, P_{2 m}\right)=s+m-1$ for $s<m-1$.

Theorem 6 For positive integers $k, m, s, t$,

(i) $R\left((k-1) K_{2}, P_{k}, P_{k}\right)=3 k-4$ if $k$ is even,

(ii) $R\left(t K_{2}, P_{2 s+1}, P_{2 m}\right)=s+m+2 t-2$ for $s<m-1<2 s+1$ and $t \geq m+s-1$.

Proof (i) Corollary 1 implies that $R\left(t K_{2}, P_{k}, P_{k}\right) \leq 2 b+t-1$ for $t \leq b$ and $b=$ $b\left(P_{k}, P_{k}\right)$. Theorem 5 (that is $b=b\left(P_{k}, P_{k}\right)=k-1$ for even $k$ ) completes the proof for the upper bound.

It is known that $R=R\left((k-1) K_{2}, P_{k}\right)=2 k+\lfloor k / 2\rfloor-3$ (see [9]). Consider the graph $G=K_{R-1} \cup K_{k / 2-1}$. Clearly $K_{R-1}$ has a blue/red coloring such that $K_{R-1}^{b}$ contains no copy of $(k-1) K_{2}$ and $K_{R-1}^{r}$ contains no copy of $P_{k}$. We can take this 
coloring and we color the edges of $K_{k / 2-1}$ with red. The edges of $\bar{G}$ we color with green. Hence $G^{g}$ contains no copy of $P_{k}$. This gives the desired lower bound.

(ii) As before, by Corollary 1 , we have $R\left(t K_{2}, P_{2 s+1}, P_{2 m}\right) \leq b+2 t-1$ for $t \geq$ $m+s-1$. On the other hand by Theorem 5 , for $s<m-1, b\left(P_{2 s+1}, P_{2 m}\right)=s+m-1$. So for $s<m-1$ and $t \geq m+s-1, R\left(t K_{2}, P_{2 s+1}, P_{2 m}\right) \leq s+m+2 t-2$.

Now, let $G=K_{R-1} \cup K_{m-1}$ where $R=R\left(t K_{2}, P_{2 s+1}\right)=2 t+\lfloor(2 s+1) / 2\rfloor-1$ for $t>\lfloor(2 s+1) / 2\rfloor$ (see [9]). Clearly $K_{R-1}$ can be colored in such a way that $K_{R-1}^{b}$ contains no copy of $t K_{2}$ and $K_{R-1}^{r}$ contains no copy of $P_{2 s+1}$. We color the subgraph $K_{m-1}$ with red and the edges of $\bar{G}$ with green. Then $G^{g}$ contains no copy of $P_{2 m}$ and the proof is complete.

\section{3 $R\left(C_{n}, k K_{2}, t K_{2}\right)$ and $R\left(P_{n}, k K_{2}, t K_{2}\right)$ for Large $n$}

Lemma 1 [3] For positive integers $m$ and $n$,

$$
b\left(m K_{2}, n K_{2}\right)=m+n-1 .
$$

Theorem $7 R\left(C_{n}, k K_{2}, t K_{2}\right)=n+k+t-2$, for sufficiently large $n$.

Proof Theorems 2, 3 and Lemma 1 give us the desired upper bound.

In [9] it is shown that $R\left(C_{n}, k K_{2}\right)=n+k-1$ for $k \leq\left\lfloor\frac{n}{2}\right\rfloor$. Consider the graph $G=K_{n+k-2} \cup \bar{K}_{t-1}$. There is a blue/red coloring of $K_{n+k-2}$ such that $G^{b}$ contains no copy of $C_{n}$ and $G^{r}$ contains no copy of $k K_{2}$. Color $\bar{G}=\bar{K}_{n+k-2}+K_{t-1}$ with color green. The theorem follows.

In [13] Maherani et al. proved that $R\left(P_{3}, k K_{2}, t K_{2}\right)=2 k+t-1$ for $k \geq t \geq 3$.

Theorem $8 R\left(P_{n}, k K_{2}, t K_{2}\right)=n+k+t-2$, for sufficiently large $n$.

Proof Clearly $R\left(P_{n}, k K_{2}, t K_{2}\right) \leq R\left(C_{n}, k K_{2}, t K_{2}\right)$. Theorem 7 gives us the upper bound.

In [9] it is shown that $R\left(P_{n}, k K_{2}\right)=n+k-1$ for $k \leq\left\lfloor\frac{n}{2}\right\rfloor$. We obtain the lower bound by considering the same coloring as in the proof of Theorem 7 .

\section{$3.4 R\left(t K_{2}, P_{3}, C_{2 n}\right)$ for $t \leq n$}

Theorem 9 [18] $R\left(P_{3}, C_{2 n}\right)=2 n$.

Theorem 10 For positive integers $t \leq n, R\left(t K_{2}, P_{3}, C_{2 n}\right)=2 n+t-1$.

Proof It is easy to see that $b\left(P_{3}, C_{2 n}\right)=n$ for $n \geq 3$. By Corollary 1 , we have $R\left(t K_{2}, C_{2 n}, P_{3}\right) \leq 2 n+t-1$ where $t \leq n$. To see the lower bound, assume that $G=K_{2 n-1} \cup \bar{K}_{t-1}$. It is clear that there is a blue/red coloring of $E\left(K_{2 n-1}\right)$ such that there is no blue copy $C_{2 n}$ and no red copy $P_{3}$. We can take this coloring and we color the edges of $\bar{G}=\bar{K}_{2 n-1}+K_{t-1}$ with color green. There is no green copy $t K_{2}$. 


\section{$3.5 R\left(t K_{2}, C_{2 m}, C_{4}\right)$}

Theorem 11 [19] $b\left(C_{2 m}, C_{4}\right)=m+1$ for $m \geq 4$.

Theorem 12 [9] $R\left(t K_{2}, C_{n}\right)=\max \{n+2 t-1-\lfloor n / 2\rfloor, n+t-1\}$ for $n \geq 3$.

Theorem 13 For a positive integer $m \geq 4$,

1. if $t \geq m+1$ then $R\left(t K_{2}, C_{2 m}, C_{4}\right)=m+2 t$,

2. if $t \leq m$ then $2 m+t \leq R\left(t K_{2}, C_{2 m}, C_{4}\right) \leq 2 m+t+1$.

Proof (1) By Corollary 1 and Theorem 11, we have $R\left(t K_{2}, C_{2 m}, C_{4}\right) \leq b+2 t-1=$ $m+2 t$ where $b=b\left(C_{2 m}, K_{2,2}\right)$ and $t \geq m+1$. To see the lower bound, consider the graph $G=K_{R-1} \cup K_{1}$ where $R=R\left(t K_{2}, C_{2 m}\right)=m+2 t-1$ for $t \geq m+1$. It is clear that there is a blue/red coloring of $E\left(K_{R-1}\right)$ such that there is no blue copy of $t K_{2}$ and no red copy of $C_{2 m}$. We can take this coloring. We color the edges of $\bar{G}=\bar{K}_{R-1}+\bar{K}_{1}$ by green. So there is no green copy of $C_{4}$.

(2) By Corollary 1 , we have $R\left(t K_{2}, C_{2 m}, C_{4}\right) \leq 2 b+t-1$ where $b=b\left(C_{2 m}, K_{2,2}\right)$ and $t \leq m+1$. By Theorem 11 , we obtain $R\left(t K_{2}, C_{2 m}, C_{4}\right) \leq 2 m+t+1$. To see the lower bound, consider the graph $G=K_{R-1} \cup K_{1}$ where $R=R\left(t K_{2}, C_{2 m}\right)=$ $2 m+t-1$. It is clear that there is a blue/red coloring of $E\left(K_{R-1}\right)$ such that there is no blue copy of $t K_{2}$ and no red copy $C_{2 m}$. We can take such a coloring and we color the edges of $\bar{G}=\bar{K}_{R-1}+\bar{K}_{1}$ with green. There is no green copy of $C_{4}$ and the proof is complete.

\subsection{Multicolor Ramsey Numbers}

This last subsection contains some results for Ramsey numbers with more than 3 colors.

Theorem 14 Let $m_{1}, m_{2}, \ldots, m_{s}$ and $k_{1}, k_{2}, \ldots, k_{t}$ be positive integers and $n>n_{1}(b)$ where $b=b\left(K_{1, k_{1}}, K_{1, k_{2}}, \ldots, K_{1, k_{t}}, m_{1} K_{2}, m_{2} K_{2}, \ldots, m_{s} K_{2}\right)$. Then

$$
R\left(C_{n}, K_{1, k_{1}}, K_{1, k_{2}}, \ldots, K_{1, k_{t}}, m_{1} K_{2}, m_{2} K_{2}, \ldots, m_{s} K_{2}\right) \leq n+b-1 .
$$

Proof By using the same argument as in Theorem 2, we have $R\left(C_{n}, K_{1, k_{1}}, K_{1, k_{2}}\right.$, $\left.\ldots, K_{1, k_{t}}, m_{1} K_{2}, m_{2} K_{2}, \ldots, m_{s} K_{2}\right) \leq R\left(C_{n}, K_{b, b}\right)$ where $b=b\left(K_{1, k_{1}}, K_{1, k_{2}}\right.$, $\left.\ldots, K_{1, k_{t}}, m_{1} K_{2}, m_{2} K_{2}, \ldots, m_{s} K_{2}\right)$. Next we apply Theorem 3 .

Lemma 2 [16] Let $m_{1}, m_{2}, \ldots, m_{s}$ and $k_{1}, k_{2}, \ldots, k_{t}$ be positive integers with $\Lambda=$ $\sum_{i=1}^{s}\left(m_{i}-1\right)$ and $\sum=\sum_{i=1}^{t}\left(k_{i}-1\right)$. Then $b\left(K_{1, k_{1}}, K_{1, k_{2}}, \ldots, K_{1, k_{t}}, m_{1} K_{2}, m_{2} K_{2}\right.$, $\left.\ldots, m_{s} K_{2}\right)=b$, where

$$
b= \begin{cases}\Lambda+1 & \text { if } \Sigma<\lfloor(\Lambda+1) / 2\rfloor, \\ \Sigma+\lfloor\Lambda / 2\rfloor+1 & \text { if } \Sigma \geq\lfloor(\Lambda+1) / 2\rfloor .\end{cases}
$$

Combining Theorem 14 and Lemma 2, we obtain the following. 
Theorem 15 Let $m_{1}, m_{2}, \ldots, m_{s}$ and $k_{1}, k_{2}, \ldots, k_{t}$ be positive integers with $\Lambda=$ $\sum_{i=1}^{s}\left(m_{i}-1\right)$ and $\sum=\sum_{i=1}^{t}\left(k_{i}-1\right)$. If $\Sigma \leq\lfloor(\Lambda+1) / 2\rfloor$ for $n>n_{1}(\Lambda)$, then

$$
R\left(C_{n}, m_{1} K_{2}, m_{2} K_{2}, \ldots, m_{s} K_{2}, K_{1, k_{1}}, K_{1, k_{2}}, \ldots, K_{1, k_{t}}\right)=n+\Lambda
$$

Proof For the upper bound, we apply Theorem 14 and Lemma 2.

For the lower bound color all edges of $G=K_{n-1} \cup \bar{K}_{\Lambda}$ by color 1 and for all edges of $\bar{G}=\bar{K}_{n-1}+K_{\Lambda}$ consider the following coloring. Color $\bar{K}_{n-1}+K_{m_{1}-1}$ and $\bar{K}_{m_{1}-1}+\bar{K}_{\sum_{i=2}^{s}\left(m_{i}-1\right)}$ by color 2 and color $\bar{K}_{n-1}+K_{m_{2}-1}$ and $\bar{K}_{m_{2}-1}+\bar{K}_{\sum_{i=3}^{s}\left(m_{i}-1\right)}$ by color 3 and in the general color $\bar{K}_{n-1}+K_{m_{j}-1}$ and $\bar{K}_{m_{j}-1}+\bar{K}_{\sum_{i=j+1}^{s}\left(m_{i}-1\right)}$ by color $j+1$ where $j \geq 1$ and finally color $\bar{K}_{n-1}+K_{m_{s}-1}$ by color $s$. Then $G^{1}$ contains no copy of $C_{n}, G^{i+1}$ contains no copy of $m_{i} K_{2}$ for $1 \leq i \leq s$, as desired. Note that this lower bound is usable on arbitrary graphs $H_{1}, \ldots, H_{t}$ instead of $K_{1, k_{1}}, K_{1, k_{2}}, \ldots, K_{1, k_{t}}$.

Corollary 4 For given positive integers $m_{1}, m_{2}, \ldots, m_{s}$ and $k_{1}, k_{2}, \ldots, k_{t}$ with $\Lambda=$ $\sum_{i=1}^{s}\left(m_{i}-1\right), \sum=\sum_{i=1}^{t}\left(k_{i}-1\right)$, sufficiently large $n$ and $\Sigma \leq\lfloor(\Lambda+1) / 2\rfloor$,

$$
R\left(P_{n}, K_{1, k_{1}}, K_{1, k_{2}}, \ldots, K_{1, k_{t}}, m_{1} K_{2}, m_{2} K_{2}, \ldots, m_{s} K_{2}\right)=n+\Lambda
$$

Lemma 3 [16] For positive integers $m, k_{1}, \ldots, k_{r} \geq 2$ and $\sum=\sum_{i=1}^{r}\left(k_{i}-1\right)$,

$$
b\left(P_{m}, K_{1, k_{1}}, \ldots, K_{1, k_{r}}\right)= \begin{cases}\Sigma+\frac{m}{2} & \text { if } \Sigma \geq \frac{m}{2}, m \text { even, } \\ \Sigma+\frac{m+1}{2} & \text { if } \Sigma \geq \frac{m-1}{2}, m \text { odd, } \Sigma \equiv 0 \bmod \left(\frac{m-1}{2}\right), \\ \Sigma+\frac{m-1}{2} & \text { if } \Sigma \geq \frac{m-1}{2}, m \text { odd, } \Sigma \neq \equiv \bmod \left(\frac{m-1}{2}\right), \\ 2 \Sigma+1 & \text { if } \frac{1}{2}\left\lfloor\frac{m}{2}\right\rfloor+1 \leq \Sigma<\left\lfloor\frac{m}{2}\right\rfloor+1, \\ \left\lfloor\frac{m+1}{2}\right\rfloor & \text { if } \Sigma<\frac{1}{2}\lfloor m / 2\rfloor .\end{cases}
$$

Theorem 16 For $\Sigma<\frac{s}{2}, m=2 s$ and $2 \leq t \leq s$,

$$
R\left(t K_{2}, P_{m}, K_{1, k_{1}}, \ldots, K_{1, k_{r}}\right)=m+t-1
$$

Proof Suppose that $\Sigma<\frac{s}{2}, m=2 s$ and $2 \leq t \leq s$. By Corollary 1 and Lemma 3, we have $R\left(t K_{2}, P_{m}, K_{1, k_{1}}, \ldots, K_{1, k_{r}}\right) \leq m+t-1$. To obtain the lower bound, divide the vertex set of $G=K_{m+t-2}$ into two parts $A$ and $B$, where $|A|=m-1$ and $|B|=t-1$. Color the edges of $G[A]$ with the second color and other edges with the first color. Note that the lower bound is usable for arbitrary graphs $H_{1}, \ldots, H_{t}$ instead of $K_{1, k_{1}}, K_{1, k_{2}}, \ldots, K_{1, k_{r}}$.

Open Access This article is distributed under the terms of the Creative Commons Attribution 4.0 International License (http://creativecommons.org/licenses/by/4.0/), which permits unrestricted use, distribution, and reproduction in any medium, provided you give appropriate credit to the original author(s) and the source, provide a link to the Creative Commons license, and indicate if changes were made. 


\section{References}

1. Bondy, J.A., Erdős, P.: Ramsey numbers for cycles in graphs. J. Comb. Theory Ser. B 14, 46-54 (1973)

2. Bielak, H.: Multicolor Ramsey numbers for some paths and cycles. Discuss. Math. Graph Theory 29, 209-218 (2009)

3. Christoua, M., Iliopoulosa, C.S., Miller, M.: Bipartite Ramsey numbers involving stars, stripes and trees. Electron. J. Graph Theory Appl. 1(2), 89-99 (2013)

4. Dzido, T.: Multicolor Ramsey numbers for paths and cycles. Discuss. Math. Graph Theory 25(1-2), 57-65 (2005)

5. Dzido, T., Fidytek, R.: On some three color Ramsey numbers for paths and cycles. Discret. Math. 309, 4955-4958 (2009)

6. Dzido, T., Kubale, M., Piwakowski, K.: On some Ramsey and Turán-type numbers for paths and cycles. Electron. J. Comb. 13, \#R55 (2006)

7. Faudree, R.J., Schelp, R.H.: Path Ramsey numbers in multicolorings. J. Comb. Theory Ser. B 19, 150-160 (1975)

8. Faudree, R.J., Schelp, R.H.: Path-path Ramsey-type numbers for the complete bipartite graph. J. Comb. Theory Ser. B 19(2), 161-173 (1975)

9. Faudree, R.J., Schelp, R.H., Sheehan, J.: Ramsey numbers for matchings. Discret. Math. 32, 105-123 (1980)

10. Gerencsér, L., Gyárfás, A.: On Ramsey-type problems. Ann. Univ. Sci. Budapestinensis Eötvös Sect. Math. Sci. 10, 167-170 (1967)

11. Gyárfás, A., Ruszinkó, M., Sárkőzy, G., Szemerédi, E.: Three-color Ramsey numbers for paths. Combinatorica 27(1), 35-69 (2007)

12. Häggkvist, R.: On the path-complete bipartite Ramsey number. Discret. Math. 75, 243-245 (1989)

13. Maherani, L., Omidi, G.R., Raeisi, G., Shahsiah, M.: On three-color Ramsey number of path. Graphs Comb. 31, 2299-2308 (2015)

14. Omidi, G.R., Raesi, G.: On multicolor Ramsey number of paths versus cycles. Electron. J. Comb. 18, \#P24 (2011)

15. Parsons, T.D.: Path-star Ramsey numbers. J. Comb. Theory Ser. B 17, 51-58 (1974)

16. Raeisi, G.: Star-path and star-stripe bipartite Ramsey numbers in multicoloring. Trans. Comb. 4(3), 37-42 (2015)

17. Ramsey, F.P.: On a problem of formal logic. Proc. Lond. Math. Soc. 30, 264-286 (1930)

18. Radziszowski, S.: Small Ramsey numbers. Electron. J. Comb. Dyn. Surv. 1, revision \#15, 1-104 (2017)

19. Zhang, R., Sun, Y.: The bipartite Ramsey numbers $b\left(C_{2 m}, K_{2,2}\right)$. Electron. J. Comb. 18(1), \#P51 (2011)

Publisher's Note Springer Nature remains neutral with regard to jurisdictional claims in published maps and institutional affiliations. 